Y. Kamoi

Nagoya Math. J.

Vol. 136 (1994), 115-131

\title{
DEFINING IDEALS OF BUCHSBAUM SEMIGROUP RINGS
}

\author{
YUUJI KAMOI
}

\section{Introduction}

Let $H$ be a simplicial semigroup. We consider the semigroup ring $k[H]$ and its defining ideal $I_{H}$. For definition see the first paragraph of Section 1.

When $\operatorname{dim}(k[H])=1$, the defining ideal $I_{H}$ of $k[H]$ has been studied by many authors (e.g. [1], [2], [8], [11], [3]). In this paper, we study the ideal $I_{H}$ using the notion of Gröbner bases for arbitrary dimension.

In [9], we gave a condition for $k[H]$ to be Cohen-Macaulay in terms of a Gröbner bases of $I_{H}$. Our aim of this paper is to extend this characterization to the case of Buchsbaum semigroup rings. We show that the Buchsbaum property of $k[H]$ is determined by the form of a Gröbner bases of $I_{H}$ in Theorem 2.6. As a corollary, we recover a result of [9] in Corollary 2.9. Also we see that if $k[H]$ is a Buchsbaum ring and not Cohen-Macaulay, then $k[H] \leq$ ht $I_{H}$ in Corollary 2.10.

We apply these results to determine Buchsbaum semigroup rings of codimension two. We can show the Gröbner bases of $I_{H}$ explicitly in Theorem 3.1.

\section{Preliminaries}

In this section, we give notations and terminologies which we shall use in this paper.

Let $\mathbf{N}$ be the set of nonnegative integers and $H$ be a finitely generated additive subsemigroup of $\mathrm{N}^{r}(r>0)$ with generators $h_{1}, \ldots, h_{r+n} \in H$ which satisfies the following conditions:

(H-1) $h_{1}, \ldots, h_{r}$ are $\mathbf{Q}$-linearly independent

$(\mathrm{H}-2)$ there exists an integer $d>0$ such that $d H \subset \sum_{t=1}^{r} \mathbf{N} h_{\imath}$.

Let $k$ be a field. We define a homomorphism $\varphi$ of polynomial rings over $k$ as:

Received October 8, 1992. 


$$
\begin{aligned}
& \varphi: S=k\left[X_{1}, \ldots, X_{r}, Y_{1}, \ldots, Y_{n}\right] \rightarrow k\left[t_{1}, \ldots, t_{r}\right] \\
& X_{i} \quad \mapsto \quad t^{h_{i}} \quad(1 \leq i \leq r) \\
& Y_{j} \quad \mapsto \quad t^{h_{r+j}} \quad(1 \leq j \leq n)
\end{aligned}
$$

where we denote $t^{h}:=t_{1}^{a_{1}} \ldots t_{r}^{a_{r}}$ for $h=\left(a_{1}, \ldots, a_{r}\right) \in \mathbf{N}^{r}$.

We put $k[H]=\operatorname{Im}(\varphi)$ and $I_{H}=\operatorname{ker}(\varphi)$. We denote

$$
\begin{aligned}
& x_{i}=t^{h_{i}}(1 \leq i \leq r) \\
& y_{j}=t^{h_{r+j}}(1 \leq j \leq n) \\
& \mathbf{m}=\left(x_{1}, \ldots, x_{r}, y_{1}, \ldots, y_{n}\right) \subset k[H] .
\end{aligned}
$$

Note that $\left\{x_{1}, \ldots, x_{r}\right\}$ is a homogeneous system of parameters of $k[H]$ by $(\mathrm{H}-1)$ and (H-2). Hence we have $r=\operatorname{dim} k[H]$ and $n=$ ht $I_{H}$.

Definition 1.1. For $\alpha, \beta \in \mathbf{N}^{m}$, we define

(1) $\alpha_{(i)}=$ the $i$-th coordinate of $\alpha$

(2) $\alpha \leq \beta \Leftrightarrow \alpha_{(i)} \leq \beta_{(i)}$ for $1 \leq i \leq n$

(3) $\alpha<\beta \Leftrightarrow \alpha \leq \beta$ for $\alpha \neq \beta$

(4) $\alpha \pm \beta=\left(\alpha_{(1)} \pm \beta_{(1)}, \ldots, \alpha_{(m)} \pm \beta_{(m)}\right)$.

We denote a monomial of $S$ by $X^{\alpha} Y^{\beta}=X_{1}^{\alpha_{(1)}} \cdots X_{1}^{\alpha_{(r)}} Y_{1}^{\beta_{(1)}} \cdots Y_{n}^{\beta_{(n)}}$ for $\alpha \in$ $\mathbf{N}^{r}, \beta \in \mathbf{N}^{n}$ and the set of all monomials of $S$ by $M_{H}$.

Definition 1.2. A total order $>_{S}$ on $M_{H}$ is called a monomial order on $S$ if it satisfies the following conditions, for every $u, v, w \in M_{H}$,

$$
\begin{aligned}
& \text { if } u<_{s} v \text {, then } u w<_{s} v w \\
& \text { if } 1 \neq u \text {, then } 1<_{s} u \text {. }
\end{aligned}
$$

Remark 1.3. It is well known that a monomial order $>_{s}$ on $S$ satisfies the following.

(1) If $(\alpha, \beta)<(\gamma, \delta)$ (in $\left.\mathbf{N}^{\gamma+n}\right)$, then $X^{\alpha} Y^{\beta}<_{s} X^{\gamma} Y^{\delta}$.

(2) Every descending sequence of monomials (w.r.t. $>_{s}$ ) is stationary. In particular, any nonempty subset of $M_{H}$ has the smallest element.

For $0 \neq f \in S$, we denote the maximal term of $f$ w.r.t. $<_{s}$ by in $(f)$ and call it the initial term of $f$. For a subset $F \subset S$, we set

$$
\text { in }(F)=\{\operatorname{in}(f) \mid 0 \neq f \in F\} .
$$


Definition 1.4. Let $I$ be an ideal of $S$ and $F$ be a finite subset of $I \backslash\{0\}$.

We call $F$ a Gröbner bases of $I$, if $(\operatorname{in}(I))=($ in $(F))$. A Gröbner bases $F$ of $I$ is called minimal, if $\operatorname{in}(F)$ is a minimal basis of (in $(I))$.

In this case, $I$ is generated by $F$ (cf. [4], [10]).

Throughout this paper, we fix a monomial order $<_{S}$ on $S$ defined as follows.

Definition 1.5. For $X^{\alpha} Y^{\beta} \in M_{H}$, we denote the total degree of $\varphi\left(X^{\alpha} Y^{\beta}\right)$ by $\operatorname{wd}\left(X^{\alpha} Y^{\beta}\right)$. We define

$$
X^{\alpha} Y^{\beta}>_{S} X^{\gamma} Y^{\delta} \Leftrightarrow\left\{\begin{array}{l}
\mathrm{wd}\left(X^{\alpha} Y^{\beta}\right)>\mathrm{wd}\left(X^{\gamma} Y^{\delta}\right) \\
\operatorname{or} \\
\mathrm{wd}\left(X^{\alpha} Y^{\beta}\right)=\mathrm{wd}\left(X^{\gamma} Y^{\delta}\right) \text { and the first non zero coordinate } \\
\text { of }(\alpha, \beta)-(\gamma, \delta)\left(\in \mathbf{Z}^{\gamma+n}\right) \text { is a negative. }
\end{array}\right.
$$

In this case, the monomial order $>_{s}$ has the following property. If $X^{\alpha} Y^{\beta}-$ $X^{\gamma} Y^{\delta} \in I_{H}$ and $X^{\alpha} Y^{\beta}>{ }_{S} X^{\gamma} Y^{\delta}$, then $\alpha>0$ implies $\gamma>0$ since $\varphi\left(X^{\alpha} Y^{\beta}\right)=$ $\varphi\left(X^{\gamma} Y^{\delta}\right)$. We shall use this fact freely this paper.

Next we define some notation.

Notation 1.6. (1) For a subset $J$ of $k[H]$, we put

$$
M(J)=\left\{X^{\alpha} Y^{\beta} \in M_{H} \mid \varphi\left(X^{\alpha} Y^{\beta}\right) \in J\right\} .
$$

(2) For $X^{\alpha} Y^{\beta} \in M_{H}$, we put

$$
\sum\left(X^{\alpha} Y^{\beta}\right)=\left\{X^{\gamma} Y^{\delta} \in M_{H} \mid \varphi\left(X^{\alpha} Y^{\beta}\right)=\varphi\left(X^{\gamma} Y^{\delta}\right), X^{\alpha} Y^{\beta}>_{S} X^{\gamma} Y^{\delta}\right\} .
$$

Remark 1.7. By definition, we have the following.

(1) For $X^{\alpha} Y^{\beta}, X^{\gamma} Y^{\delta} \in M_{H}$ with $X^{\alpha} Y^{\beta} \neq X^{\gamma} Y^{\delta}, X^{\alpha} Y^{\beta}-X^{\gamma} Y^{\delta} \in I_{H}$ if and only if $X^{\alpha} Y^{\beta} \in \sum\left(X^{\gamma} Y^{\delta}\right)$ or $X^{\gamma} Y^{\delta} \in \sum\left(X^{\alpha} Y^{\beta}\right)$.

(2) For $X^{\alpha} Y^{\beta} \in M_{H}, \sum\left(X^{\alpha} Y^{\beta}\right) \neq \phi$ if and only if $X^{\alpha} Y^{\beta} \in\left(\right.$ in $\left.\left(I_{H}\right)\right)$.

(3) If $X^{\alpha} Y^{\beta}$ is the smallest element of $\sum\left(X^{\gamma} Y^{\delta}\right)$ (w.r.t. $>_{S}$ ), then $\sum\left(X^{\alpha} Y^{\beta}\right)=\phi$.

(4) If $(\alpha, \beta) \leq(\gamma, \delta)$ (in $\left.\mathbf{N}^{\gamma+n}\right)$, then $\sum\left(X^{\alpha} Y^{\beta}\right) \neq \phi$ implies $\sum\left(X^{\gamma} Y^{\delta}\right) \neq \phi$.

(5) If $X^{\alpha} Y^{\beta}-X^{\gamma} Y^{\delta} \in I_{H}$ and $J \subset k[H]$, then $X^{\alpha} Y^{\beta} \in M(J)$ if and only if $X^{\gamma} Y^{\delta} \in M(J)$.

(6) For $1 \leq i \leq r, X^{\alpha} Y^{\beta} \in M\left(\left(x_{\imath}\right)\right)$ if and only if there exists $X^{\alpha} Y^{\beta}-$ $X_{i} X^{\gamma} Y^{\delta} \in I_{H}$. 
(7) For $1 \leq i \leq r$, if $Y^{\beta} \in M\left(\left(x_{i}\right)\right)$, then $\sum\left(Y^{\beta}\right) \neq \phi$.

We put

$$
\mathscr{R}=\left\{X^{\alpha} Y^{\beta}-X^{\gamma} Y^{\delta} \in S \mid X^{\gamma} Y^{\delta} \in \sum\left(X^{\alpha} Y^{\beta}\right) \text { and } \operatorname{Gcd}\left(X^{\alpha} Y^{\beta}, X^{\gamma} Y^{\delta}\right)=1\right\} \text {. }
$$

Then, by Remark 1.7 (1), we have $\mathscr{R} \subset I_{H}$. Furthermore, the following result is standard (cf. Proposition 1.4 and Proposition 1.5 of [8]).

Proposition 1.8. We have $I_{H}=(\mathscr{R})$ and $\left(\operatorname{in}\left(I_{H}\right)\right)=($ in $(\mathscr{R}))$. Thus we can choose a Gröbner bases of $I_{H}$ from $\mathscr{R}$.

\section{Buchsbaum property of semigroup rings}

In this section, we give a condition for $k[H]$ to be Buchsbaum in terms of a Gröbner bases of $I_{H}$.

We recall that a Noetherian local ring $(A, \mathbf{n})$ is called a Buchsbaum ring, if $l_{A}(A / \mathrm{q})-e_{\mathbf{q}}(A)$ is a constant for every parameter ideal $\mathbf{q}$ of $A$.

$k[H]$ is called a Buchsbaum ring, if the local ring $k[H]_{\mathbf{m}}$ of $k[H]$ at $\mathbf{m}$ is a Buchsbaum ring. In this case, $k[H]$ satisfies the following conditions: for every $1 \leq i<j \leq r$,

$$
\begin{gathered}
{\left[\left(x_{1}^{n_{1}}, \ldots, x_{\imath-1}^{n_{i-1}}\right): x_{i}^{n_{i}}\right]=\left[\left(x_{1}^{n_{1}}, \ldots, x_{i-1}^{n_{i-1}}\right): \mathbf{m}\right]} \\
{\left[\left(x_{1}^{n_{1}}, \ldots, x_{i-1}^{n_{i-1}}\right): x_{i}^{n_{i}} x_{j}^{n_{j}}\right]=\left[\left(x_{1}^{n_{1}}, \ldots, x_{1-1}^{n_{i-1}}\right): x_{i}^{n_{i}}\right]}
\end{gathered}
$$

where $n_{1}, \ldots, n_{r} \in \mathbf{N} \backslash\{0\}$ (cf. Proposition 1.10 of ch. 1 in [12]).

In [6], S. Goto proved the following criterion for $k[H]$ to be Buchsbaum.

THEOREM 2.1 (Theorem 3.1 in [6]). The following conditions are equivalent.

(1) $k[H]$ is a Buchsbaum ring.

(2) There exists a simplicial semigroup $H^{\prime} \subset \mathbf{N}^{r}$ such that $k\left[H^{\prime}\right]$ is a Cohen-Macaulay and $\mathbf{m} k\left[H^{\prime}\right] \subset k[H]$.

(3) For $1 \leq i \leq r,\left[\left(x_{1}^{2}, \ldots, x_{i-1}^{2}\right): x_{\imath}^{2}\right]=\left[\left(x_{1}^{2}, \ldots, x_{i-1}^{2}\right): \mathbf{m}\right]$ (i.e. $k[H]$ is a quasi-Buchsbaum ring).

Lemma 2.2. For $t^{v}, t^{u_{1}}, \ldots, t^{u_{p}} \in k[H]$, we have

$$
\left[\left(t^{u_{1}}, \ldots, t^{u_{p}}\right): t^{v}\right]=\sum_{i=1}^{p}\left[\left(t^{u_{i}}\right): t^{v}\right]
$$


Proof. It is clear that $\left[\left(t^{u_{1}}, \ldots, t^{u_{p}}\right): t^{v}\right] \supset \sum_{l=1}^{p}\left[\left(t^{u_{i}}\right): t^{v}\right]$. We show the converse inclusion.

For $f \in\left[\left(t^{u_{1}}, \ldots, t^{u_{p}}\right): t^{v}\right]$, we write $f=\sum_{i=1}^{m} c_{t} t^{w_{i}}, c_{i} \neq 0$ and $w_{\imath} \in H(1 \leq$ $i \leq m)$. Then $t^{v} f=\sum_{i=1}^{m} c_{t} t^{v+w_{i}} \in\left(t^{u_{1}}, \ldots, t^{u_{p}}\right)$. Since $\left(t^{u_{1}}, \ldots, t^{u_{p}}\right)$ is a $\mathbf{N}^{r}$-graded ideal, $t^{v+w_{j}} \in\left(t^{u_{1}}, \ldots, t^{u_{p}}\right)$ for every $1 \leq j \leq m$. Then we have $t^{v+w_{j}}=t^{h+u_{i}}$ for some $1 \leq i \leq p$ and for some $h \in H$. Thus $t^{w_{j}} \in\left[\left(t^{u_{i}}\right): t^{v}\right]$ and $f \in \sum_{i=1}^{p}\left[\left(t^{u_{i}}\right)\right.$ : $\left.t^{v}\right]$.

Hence we have the following by Theorem 2.1 and Lemma 2.2 .

Proposition 2.3. The following conditions are equivalent.

(1) $k[H]$ is a Buchsbaum ring.

(2) For every $1 \leq i<j \leq r,\left[\left(x_{i}^{2}\right): x_{j}^{2}\right]=\left[\left(x_{i}^{2}\right): \mathbf{m}\right]$.

(2') For $1 \leq i<j \leq r$ and $u, v \in H$, if $2 h_{j}+v=2 h_{i}+u$, when $(H \backslash\{0\})$ $+v \subset H+2 h_{\imath}$.

Proposition 2.4. (Theorem 2.6 in [7]). The following conditions are equivalent.

(1) $k[H]$ is a Cohen-Macaulay ring.

(2) $x_{1}, \ldots, x_{r}$ are regular sequence of $k[H]$.

(3) $\left[\left(x_{i}\right): x_{j}\right]=\left(x_{i}\right)$ for every $1 \leq i<j \leq r$.

(3') For $1 \leq i<j \leq r$ and $u, v \in H$, if $h_{j}+v=h_{i}+u$, then $v \in H+h_{i}$.

We define the subsets $\mathscr{R}_{H}, \mathscr{F}_{H}$ and $\mathscr{F}^{\prime}$ of $\mathscr{R}$ as:

$$
\begin{aligned}
\mathscr{R}_{H} & =\left\{X^{\alpha} Y^{\beta}-X^{\gamma} Y^{\delta} \in \mathscr{R} \mid \sum\left(Y^{\delta}\right)=\phi\right\} \\
\mathscr{F}_{H} & =\left\{f \in \mathscr{R}_{H} \mid \operatorname{in}(f)=Y^{\beta}, \beta \in \mathbf{N}^{n}\right\} \\
\mathscr{F}^{\prime} & =\left\{f \in \mathscr{R}_{H} \mid \operatorname{in}(f) \in\left(\operatorname{in}\left(\mathscr{F}_{H}\right)\right)\right\} .
\end{aligned}
$$

By Proposition 1.8, Remark 1.7 (3) and (4), it is easy to see that $\left(\operatorname{in}\left(I_{H}\right)\right)=$ $\left(\right.$ in $\left(\mathscr{R}_{H}\right)$ ).

Definition 2.5. A sequence of monomials $\left(Y^{\beta_{1}}, \ldots, Y^{\beta_{r}}\right)$ is called a $\mathrm{B}$-sequence, if it satisfies the following conditions: for every $1 \leq i \neq j \leq r$,

$$
\begin{aligned}
& \text { (B-1) } \sum\left(Y^{\beta_{i}}\right)=\phi \\
& \text { (B-2) } Y^{\beta_{i}} \in M\left(\left[\left(x_{i}\right): \mathbf{m}\right]\right) \\
& \text { (B-3) } Y^{\beta_{i}} \notin M\left(\left[\left(x_{j}\right): \mathbf{m}\right]\right) \\
& \text { (B-4) } \operatorname{Gcd}\left(Y^{\beta_{i}}, Y^{\beta_{i}}\right)=1
\end{aligned}
$$




$$
\text { (B-5) } X_{j} Y^{\beta_{i}}-X_{i} Y^{\beta_{j}} \in I_{H} \text {. }
$$

We denote by $\Delta_{H}$ the set of all B-sequences and put

$$
\mathscr{G}_{H}=\left\{X_{j} Y^{\beta_{i}}-X_{i} Y^{\beta_{j}} \mid\left(Y^{\beta_{1}}, \ldots, Y^{\beta_{r}}\right) \in \Delta_{H}, 1 \leq i<j \leq r\right\} .
$$

The main purpose of this section is to prove the following result.

THEOREM 2.6. The following conditions are equivalent.

(1) $k[H]$ is a Buchsbaum ring.

(2) We can choose a Gröbner bases of $I_{H}$ from $\mathscr{F}_{H} \cup \mathscr{G}_{H}\left(\right.$ or $\left(\right.$ in $\left.\left(I_{H}\right)\right)=\left(\right.$ in $\left(\mathscr{F}_{H}\right.$ $\left.\left.\cup \mathscr{G}_{H}\right)\right)$ ).

To prove our result, we need some lemmas.

Lemma 2.7. Suppose that $k[H]$ is a Buchsbaum ring.

(1) If $X^{\alpha} Y^{\beta} \in M\left(\left[\left(x_{i}\right): x_{j}\right]\right)$ and $\alpha_{(i)}=0$, then $Y^{\beta} \in M\left(\left[\left(x_{i}\right): x_{j}\right]\right)$.

(2) If $Y^{\beta} \in M\left(\left[\left(x_{i}\right): x_{j}\right]\right)$ and $\sum\left(Y^{\beta}\right)=\phi$, then there exists $\left(Y^{\beta_{1}}, \ldots, Y^{\beta_{r}}\right) \in$ $\Delta_{H}$ such that $\beta=\beta_{i}$.

Proof. (1) This is proved by induction on the degree of $X^{\alpha}$. If $\alpha=0$, then there is nothing to prove. If $\alpha>0$, then we can find $1 \leq l \leq r, l \neq i$ such that $X^{\alpha}=X_{l} X^{\alpha^{\prime}}$. Then we have $X^{\alpha^{\prime}} Y^{\beta} \in M\left(\left[\left(x_{i}\right): x_{j} x_{l}\right]\right)$. Since $k[H]$ is Buchsbaum, $\left[\left(x_{i}\right): x_{j} x_{l}\right]=\left[\left(x_{i}\right): x_{j}\right]$. Hence, by the induction hypothesis, we have $Y^{\beta} \in$ $M\left(\left[\left(x_{i}\right): x_{j}\right]\right)$.

(2) Since $k[H]$ is Buchsbaum, $\left[\left(x_{i}\right): x_{j}\right]=\left[\left(x_{i}\right): \mathbf{m}\right]$. Then, by Remark 1.7 (6), we have $X_{k} Y^{\beta}-X_{i} X^{\alpha_{k}} Y^{\beta_{k}} \in I_{H}$ for $1 \leq k \neq i \leq r$. If $\sum\left(X^{\alpha_{k}} Y^{\beta_{k}}\right) \neq \phi$, then we can replace $X^{\alpha_{k}} Y^{\beta_{k}}$ by the smallest element of $\sum\left(X^{\alpha_{k}} Y^{\beta_{k}}\right)$. Therefore we may assume $\sum\left(X^{\alpha_{k}} Y^{\beta_{k}}\right)=\phi$. If $\alpha_{k(k)}>0$, then $Y^{\beta} \in M\left(\left(x_{i}\right)\right)$ and, by Remark 1.7 (7), $\sum\left(Y^{\beta}\right) \neq \phi$. This contradicts our assumption. Thus $\alpha_{k(k)}=0$. On the other hand, $X^{\alpha_{k}} Y^{\beta_{k}} \in M\left(\left[\left(x_{k}\right): x_{i}\right]\right)$. By (1), this implies $Y^{\beta_{k}} \in\left(\left[\left(x_{k}\right): x_{i}\right]\right)$. Then, by Remark 1.7 (6), there exists $X_{i} Y^{\beta_{k}}-X_{k} X^{\gamma} Y^{\delta} \in I_{H}$. Hence we have

$$
\left(X_{k} Y^{\beta}-X_{i} X^{\alpha_{k}} Y^{\beta_{k}}\right)-X^{\alpha_{k}}\left(X_{i} Y^{\beta_{k}}-X_{k} X^{\gamma} Y^{\delta}\right)=X_{k}\left(Y^{\beta}-X^{\alpha_{k}+\gamma} Y^{\delta}\right) \in I_{H}
$$

and $Y^{\beta}-X^{\alpha_{k}+\gamma} Y^{\delta} \in I_{H}$, since $I_{H}$ is a prime ideal. By Remark 1.7 (1) and $\sum\left(Y^{\beta}\right)=\phi$, we have $Y^{\beta} \leq_{S} X^{\alpha_{k}+\gamma} Y^{\delta}$ and, by the definition of the ordering $>_{s}$, $\alpha_{k}+\gamma=0$. Hence we have $X_{k} Y^{\beta}-X_{i} Y^{\beta_{k}} \in I_{H}$ with $\sum\left(Y^{\beta_{k}}\right)=\phi$ for $1 \leq k \neq i$ $\leq r$, We put $\beta_{i}=\beta$. Then the sequence $\left(Y^{\beta_{1}}, \ldots, Y^{\beta_{r}}\right)$ satisfies the conditions $(\mathrm{B}-1)$ and (B-2) of Definition 2.5. We show the other conditions are also satisfied. 
(B-5): For every $1 \leq k<l \leq r$, we have the following relation

$$
X_{k}\left(X_{l} Y^{\beta_{i}}-X_{\imath} Y^{\beta_{l}}\right)-X_{l}\left(X_{k} Y^{\beta_{i}}-X_{\imath} Y^{\beta_{k}}\right)=X_{i}\left(X_{l} Y^{\beta_{k}}-X_{k} Y^{\beta_{l}}\right) \in I_{H} .
$$

Since $I_{H}$ is a prime ideal, we have $X_{l} Y^{\beta_{k}}-X_{k} Y^{\beta_{l}} \in I_{H}$.

(B-3): For some $1 \leq k, l \leq r$, if $Y^{\beta_{k}} \in M\left(\left[\left(x_{l}\right): \mathbf{m}\right]\right)$, then there exists $X_{k} Y^{\beta_{k}}-X_{l} X^{\gamma} Y^{\delta} \in I_{H}$. Hence we have the relation

$$
X_{l}\left(X_{k} Y^{\beta_{k}}-X_{l} X^{\gamma} Y^{\delta}\right)-X_{k}\left(X_{l} Y^{\beta_{k}}-X_{k} Y^{\beta_{l}}\right)=X_{k}^{2} Y^{\beta_{l}}-X_{l}^{2} X^{\gamma} Y^{\delta} \in I_{H}
$$

and $Y^{\beta_{l}} \in M\left(\left[\left(x_{l}^{2}\right): x_{k}^{2}\right]\right)$. Since $k[H]$ is Buchsbaum, $\left[\left(x_{l}^{2}\right): x_{k}^{2}\right]=\left[\left(x_{l}^{2}\right): x_{k}\right]$ and $\left[\left(x_{k}\right): x_{l}^{2}\right]=\left[\left(x_{k}\right): x_{l}\right]$. Then it is easy to see that $\left[\left(x_{l}^{2}\right): x_{k}^{2}\right]=x_{l}\left[\left(x_{l}\right): x_{k}\right]$ and thus $Y^{\beta_{l}} \in M\left(\left(x_{l}\right)\right)$. Hence, by Remark $1.7(7), \sum\left(Y^{\beta_{l}}\right) \neq \phi$. This contradicts condition (B-1). Thus $Y^{\beta_{k}} \notin M\left(\left[\left(x_{l}\right): \mathbf{m}\right]\right)$.

(B-4): For some $1 \leq k, l \leq r$, if $\operatorname{Gcd}\left(Y^{\beta_{k}}, Y^{\beta_{l}}\right) \neq 1$, then we can write

$$
X_{l} Y^{\beta_{k}}-X_{k} Y^{\beta_{l}}=Y^{\delta}\left(X_{l} Y^{\delta_{k}}-X_{k} Y^{\delta_{l}}\right) \in I_{H}
$$

where $Y^{\delta}=\operatorname{Gcd}\left(Y^{\beta_{k}}, Y^{\beta_{l}}\right), \delta_{k}=\beta_{k}-\delta$ and $\delta_{l}=\beta_{l}-\delta$. Then we have $X_{l} Y^{\delta_{k}}$ $-X_{k} Y^{\delta_{l}} \in I_{H} \quad$ and $\quad Y^{\delta_{k}} \in M\left(\left[\left(x_{k}\right): x_{l}\right]\right)=M\left(\left[\left(x_{k}\right): \mathbf{m}\right]\right)$, since $k[H]$ is Buchsbaum. Since $Y^{\delta} \neq 1$, we have $Y^{\beta_{k}}=Y^{\delta+\delta_{k}} \in M\left(\left(x_{k}\right)\right)$. Then, by (1.7), 7), $\sum\left(Y^{\beta_{k}}\right) \neq \phi$. This is a contradiction. Hence $\operatorname{Gcd}\left(Y^{\beta_{k}}, Y^{\beta_{l}}\right)=1$.

Lemma 2.8. Suppose that $\left(\operatorname{in}\left(I_{H}\right)\right)=\left(\operatorname{in}\left(\mathscr{F}_{H} \cup \mathscr{G}_{H}\right)\right)$.

(1) For $X^{\alpha} Y^{\beta}-X^{\gamma} Y^{\delta} \in I_{H}$ with $\sum\left(Y^{\beta}\right)=\phi$ and $X^{\alpha} Y^{\beta}>_{S} X^{\gamma} Y^{\delta}$, there exists $\left(Y^{\beta_{1}}, \ldots, Y^{\beta_{r}}\right) \in \Delta_{H}$ such that $X^{\alpha} Y^{\beta}=X_{j} X^{\alpha^{\prime}} Y^{\beta_{i}}$ for some $1 \leq i \neq j$ $\leq r$.

(2) $\mathscr{R}_{H}=\mathscr{F}^{\prime} \cup \mathscr{G}_{H}$.

Proof. (1) Since $\left(\operatorname{in}\left(I_{H}\right)\right)=\left(\operatorname{in}\left(\mathscr{F}_{H} \cup \mathscr{G}_{H}\right)\right)$ and $\sum\left(Y^{\beta}\right)=\phi$, we have $X^{\alpha} Y^{\beta}$ $\in\left(\right.$ in $\left(\mathscr{G}_{H}\right)$ ) by Remark 1.7 (2) and (4). Thus there exists a B-sequence $\left(Y^{\beta_{1}}, \ldots, Y^{\beta_{r}}\right)$ and an element $X_{j} Y^{\beta_{i}}-X_{i} Y^{\beta_{j}} \in \mathscr{G}_{H}$ such that $X^{\alpha} Y^{\beta}$ is divisible by $X_{j} Y^{\beta^{2}}$. If $\beta_{\imath}<\beta$, then $Y^{\beta} \in M\left(\left(x_{i}\right)\right)$ by the condition (B-2). By Remark 1.7 (7), this contradicts $\sum\left(Y^{\beta}\right)=\phi$. Hence we have $\beta=\beta_{i}$.

(2) Suppose that $\mathscr{R}_{H} \neq \mathscr{F}^{\prime} \cup \mathscr{G}_{H}$. Then, by Remark 1.3 (2), there exists $f=$ $X^{\alpha} Y^{\beta}-X^{\gamma} Y^{\delta} \in \mathscr{R}_{H} \backslash \mathscr{F}^{\prime} \cup \mathscr{G}_{H}$ such that in $(f)$ is smallest element of in $\left(\mathscr{R}_{H} \backslash \mathscr{F}^{\prime}\right.$ $\left.\cup \mathscr{G}_{H}\right)$. Since $f \notin \mathscr{F}^{\prime}$ and Remark 1.7 (2), we have $\sum\left(Y^{\beta}\right)=\phi$ and, by (1), there exists $g=X_{j} Y^{\beta_{i}}-X_{i} Y^{\beta_{j}} \in \mathscr{G}_{H}$ such that $X^{\alpha} Y^{\beta}=X_{j} X^{\alpha^{\prime}} Y^{\beta_{i}}$. Thus we have

$$
f-X^{\alpha^{\prime}} g=X_{i} X^{\alpha^{\prime}} Y^{\beta_{j}}-X^{\gamma} Y^{\delta} \in I_{H} .
$$

If $X_{t} X^{\alpha^{\prime}} Y^{\beta_{j}}=X^{\gamma} Y^{\delta}$, then $X^{\alpha^{\prime}}=1$, since $\operatorname{Gcd}\left(X^{\alpha}, X^{\gamma}\right)=1$. Thus $f=g \in \mathscr{G}_{H}$. 
This is a contradiction. Hence we have $X_{i} X^{\alpha^{\prime}} Y^{\beta_{j}} \neq X^{\gamma} Y^{\delta}$. We put

$$
X^{\mu} Y^{\nu}=\operatorname{Gcd}\left(X_{i} X^{\alpha^{\prime}} Y^{\beta_{j}}, X^{\gamma} Y^{\delta}\right) \text { and } g^{\prime}=X^{\mu_{1}} Y^{\nu_{1}}-X^{\mu_{2}} Y^{\nu_{2}} \in I_{H}
$$

where $X_{i} X^{\alpha^{\prime}} Y^{\beta_{j}}=X^{\mu+\mu_{1}} Y^{\nu+\nu_{1}}$ and $X^{\gamma} Y^{\delta}=X^{\mu+\mu_{2}} Y^{\nu+\nu_{2}}$. Then either $g^{\prime} \in \mathscr{R}_{H}$ or $-g^{\prime} \in \mathscr{R}_{H}$. Since in $(f)>_{S}$ in $\left(g^{\prime}\right), g^{\prime} \in \mathscr{F}^{\prime} \cup \mathscr{G}_{H}$ or $-g^{\prime} \in \mathscr{F}^{\prime} \cup \mathscr{G}_{H}$ by the minimality of $f$. We note that $\Sigma\left(Y^{\beta_{j}}\right)=\phi$ and $\Sigma\left(Y^{\delta}\right)=\phi$. This implies that $\sum\left(Y^{\nu_{p}}\right)=\phi(p=1,2)$ and $\pm g^{\prime} \notin \mathscr{F}^{\prime}$. Hence we have either $g^{\prime} \in \mathscr{G}_{H}$ or $-g^{\prime} \in$ $\mathscr{G}_{H}$ and there exists $\left(Y^{\delta_{1}}, \ldots, Y^{\delta_{r}}\right) \in \Delta_{H}$ such that $g^{\prime}=X_{l} Y^{\delta_{k}}-X_{k} Y^{\delta_{l}}$ for some $1 \leq k \neq l \leq r$. Since $\alpha_{(j)}>0, \gamma_{(k)}>0$ and $\operatorname{Gcd}\left(X^{\alpha}, X^{\gamma}\right)=1$, we have $j \neq k$. Then

$$
Y^{\beta_{j}}=Y^{\nu+\delta_{k}} \in M\left(\left[\left(x_{j}\right): \mathbf{m}\right]\right) \cap M\left(\left[\left(x_{k}\right): \mathbf{m}\right]\right) .
$$

This contradicts condition (B-3). Hence we have $\mathscr{R}_{H}=\mathscr{F}^{\prime} \cup \mathscr{G}_{H}$.

Proof of Theorem 2.6. (1) $\Rightarrow(2)$ For $f=X^{\alpha} Y^{\beta}-X^{r} Y^{\delta} \in \mathscr{R}_{H}$, we suppose that in $(f) \notin\left(\right.$ in $\left.\left(\mathscr{F}_{H}\right)\right)$. Then $\alpha>0$ and, by the definition of $>_{S}$, we can find $1 \leq i<j \leq r$ such that $f=X_{j} X^{\alpha^{\prime}} Y^{\beta}-X_{i} X^{\gamma^{\prime}} Y^{\delta}$. Thus $X^{\alpha^{\prime}} Y^{\beta} \in M\left(\left[\left(x_{i}\right)\right.\right.$ : $\left.\left.x_{j}\right]\right)$ by Remark $1.7(5)$. Since $\operatorname{Gcd}\left(X^{\alpha}, X^{\gamma}\right)=1$, we have $Y^{\beta} \in M\left(\left[\left(x_{i}\right): x_{j}\right]\right)$ by (2.7), 1). Then, by Remark 1.7 (2) and (4), $\sum\left(Y^{\beta}\right)=\phi$ and, by Lemma 2.7 (2), there exists $\left(Y^{\beta_{1}}, \ldots, Y^{\beta_{r}}\right) \in \Delta_{H}$ such that $\beta=\beta_{i}$. Thus, $X_{j} Y^{\beta_{j}}-X_{i} Y^{\beta_{j}} \in \mathscr{G}_{H}$ and $\operatorname{in}(f)=X^{\alpha} Y^{\beta} \in\left(\operatorname{in}\left(\mathscr{G}_{H}\right)\right)$.

Hence we have $\left(\operatorname{in}\left(I_{H}\right)\right)=\left(\operatorname{in}\left(\mathscr{F}_{H} \cup \mathscr{G}_{H}\right)\right)$.

$(2) \Rightarrow(1)$ By Proposition 2.3, it suffices to show that $\left[\left(x_{i}^{2}\right): x_{j}^{2}\right]=\left[\left(x_{i}^{2}\right)\right.$ : $\mathbf{m}]$ for $1 \leq i<j \leq r$. Therefore we suppose that $\left[\left(x_{i}^{2}\right): x_{j}^{2}\right] \neq\left[\left(x_{i}^{2}\right): \mathbf{m}\right]$. Then there exists the smallest element $X^{\alpha} Y^{\beta}$ of $M\left(\left[\left(x_{i}^{2}\right): x_{j}^{2}\right] \backslash\left[\left(x_{i}^{2}\right): \mathbf{m}\right]\right.$. We note that $\alpha_{(i)} \leq 1$ and $\sum\left(Y^{\beta}\right)=\phi$. By Remark $1.7(6)$, there exists $X_{j}^{2} X^{\alpha} Y^{\beta}-X_{i}^{2} X^{\gamma} Y^{\delta}$ $\in I_{H}$. If $\sum\left(X^{\gamma} Y^{\delta}\right) \neq \phi$, then we can replace $X^{\gamma} Y^{\delta}$ by the smallest element of $\sum\left(X^{\gamma} Y^{\delta}\right)$. Thus we may assume that $\sum\left(X^{\gamma} Y^{\delta}\right)=\phi$. Since $X^{\alpha} Y^{\beta} \notin M\left(\left(x_{i}^{2}\right)\right)$, we have $\gamma_{(j)} \leq 1$. Now we put

$$
X^{\mu} Y^{\nu}=\operatorname{Gcd}\left(X_{j}^{2} X^{\alpha} Y^{\beta}, X_{i}^{2} X^{\gamma} Y^{\delta}\right) \text { and } g=X^{\mu_{1}} Y^{\nu_{1}}-X^{\mu_{2}} Y^{\nu_{2}} \in I_{H}
$$

where $X_{j}^{2} X^{\alpha} Y^{\beta}=X^{\mu+\mu_{1}} Y^{\nu+\nu_{1}}$ and $X_{i}^{2} X^{\gamma} Y^{\delta}=X^{\mu+\mu_{2}} Y^{\nu+\nu_{2}}$. Then $g \in \mathscr{R}_{H}$ or $-g$ $\in \mathscr{R}_{H}$. But, by Remark 1.7 (4), $\Sigma\left(Y^{\nu_{1}}\right)=\phi=\sum\left(Y^{\nu_{2}}\right)$ and, by Remark 1.7 (2), in $(g) \notin\left(\right.$ in $\left.\left(\mathscr{F}_{H}\right)\right)$. Then, by Lemma $2.8(2)$, we have $g \in \mathscr{G}_{H}$ or $-g \in \mathscr{G}_{H}$. On the other hand, $\mu_{1(j)}>0$ and $\mu_{2(i)}>0$, since $\gamma_{(j)} \leq 1, \alpha_{(i)} \leq 1$. Thus we have $X^{\mu_{1}}=X_{j}\left(\right.$ resp. $\left.X^{\mu_{2}}=X_{i}\right)$ and $\alpha_{(i)}=1$ (resp. $\gamma_{(j)}=1$ ). Hence $X^{\alpha} Y^{\beta} \in M\left(x_{i}\right.$ $\left.\left[\left(x_{i}\right): \mathbf{m}\right]\right) \subset M\left(\left[\left(x_{i}^{2}\right): \mathbf{m}\right]\right)$. This is a contradiction. 
As a consequence of Theorem 2.6, we have the following corollaries.

Corollary 2.9 (Theorem 1.2 in [9]). The followrng conditions are equivalent.

(1) $k[H]$ is a Cohen-Macaulay ring.

(2) We can take a Gröbner bases of $I_{H}$ from $\mathscr{F}_{H}\left(\right.$ or $\left.\left(\operatorname{in}\left(I_{H}\right)\right)=\left(\operatorname{in}\left(\mathscr{F}_{H}\right)\right)\right)$.

Proof. By Theorem 2.6, we may assume that $k[H]$ is a Buchsbaum ring.

If $k[H]$ is Cohen-Macaulay, then we have $\left[\left(x_{i}\right): x_{j}\right]=\left(x_{i}\right)$ for every $1 \leq i$ $\neq j \leq r$. Hence, if $Y^{\beta} \in M\left(\left[\left(x_{i}\right): \mathbf{m}\right]\right)$, then $\sum\left(Y^{\beta}\right) \neq \phi$ by Remark 1.7 (7). Thus $\mathscr{G}_{H}=\phi$ and $\Delta_{H}=\phi$ (cf. Definition 2.5). Hence we have $\left(\operatorname{in}\left(I_{H}\right)\right)=$ (in $\left.\left(\mathscr{F}_{H}\right)\right)$.

Conversely, if $k[H]$ is not Cohen-Macaulay, then there exists $1 \leq i<j \leq r$ such that $\left[\left(x_{i}\right): x_{j}\right] \neq\left(x_{i}\right)$ (cf. Proposition 2.4). Since $k[H]$ is a Buchsbaum, we have $\left[\left(x_{1}\right): x_{1}\right]=\left[\left(x_{i}\right): \mathbf{m}\right]$ and there exists the smallest element $Y^{\beta} \in M\left(\left[\left(x_{i}\right)\right.\right.$ : $\left.x_{j}\right] \backslash\left(x_{\imath}\right)$ ) (cf. Lemma $2.7(1)$ ). Then, by Remark $1.7(2), Y^{\beta} \notin\left(\operatorname{in}\left(I_{H}\right)\right)$ and $X_{j} Y^{\beta}$ $\in\left(\right.$ in $\left.\left(I_{H}\right)\right)$ since $i<j$. This shows that $\left(\operatorname{in}\left(I_{H}\right)\right) \neq\left(\right.$ in $\left.\left(\mathscr{F}_{H}\right)\right)$.

Corollary 2.10. If $k[H]$ is a Buchsbaum ring and not Cohen-Macaulay, then we have

$$
\left.\operatorname{dim} k[H] \leq \mathrm{ht}_{S} I_{H} \text { (i.e. } r \leq n\right)
$$

Proof. Since $k[H]$ is not Cohen-Macaulay, we have $\mathscr{G}_{H} \neq \phi$ by Theorem 2.6 and Corollary 2.9. Thus there exists $\left(Y^{\beta_{1}}, \ldots, Y^{\beta_{r}}\right) \in \Delta_{H}$. We put $n_{i}=\#\{k \in$ $\left.\{1, \ldots, r\} \mid \beta_{i(k)}>0\right\}$. Then, by (B-2) of Definition $2.5, \beta_{i} \neq 0$ and $n_{i}>0$ for $1 \leq i \leq r$. Hence $r \leq \sum_{i=1}^{r} n_{i}$. On the other hand, $\sum_{t=1}^{r} n_{i} \leq n$ since $\operatorname{Gcd}\left(Y^{\beta_{k}}\right.$, $\left.Y^{\beta_{l}}\right)=1$ (cf. (B-4) of Definition 2.5), for $1 \leq k \neq l \leq r$.

In the following example, we see that there exists a Buchsbaum and not Cohen-Macaulay semigroup ring $k[H]$ with $k[H]=r$ and ht $I_{H}=n$ for $2 \leq r$ $\leq n \in \mathbf{N}$.

Example 2.11. For $2 \leq r \leq n \in \mathbf{N}$, we let $a_{0}, \ldots, a_{p} \in \mathbf{N}(p=n-r+1)$ such that $a_{p} \notin \sum_{l=0}^{p-1} \mathbf{N} a_{i}$ and $a_{p}+a_{j} \notin \sum_{i=0}^{p-1} \mathbf{N} a_{i}$, for $1 \leq j \leq p$.

(e.g. $\left(a_{0}, \ldots, a_{p-1}\right)=1, a_{p}=\max \left\{a \in \mathbf{N} \mid a \notin \sum_{i=0}^{p-1} \mathbf{N} a_{i}\right\}$, if $r<n$ or $a_{0}=$ $2, a_{1}=1$, if $r=n$.)

We put $h_{1}, \ldots, h_{r+n}, g \in \mathbf{N}^{r}$ as follows: 


$$
\begin{aligned}
& h_{i} \quad=\left(h_{i(i)}=a_{0}, h_{i}(j)=0(j \neq i)\right) \quad(1 \leq i \leq r) \\
& h_{2 r+j}=\left(a_{j}, \ldots, a_{j}\right) \quad(1 \leq j \leq p-1) \\
& g \quad=\left(a_{p}, \ldots, a_{p}\right) \\
& h_{r+i}=h_{i}+g \quad(1 \leq i \leq r) \\
& H=\sum_{i=1}^{r+n} \mathbf{N} h_{i} \quad \subset \mathbf{N}^{r} \\
& H^{\prime} \quad=\sum_{1 \leq j \leq r} \text { or }{ }_{2 \leq j \leq r+n} \mathbf{N} h_{j}+\mathbf{N} g \subset \mathbf{N}^{r} \text {. }
\end{aligned}
$$

We define

$$
\varphi: k\left[X_{1}, \ldots, X_{r}, Y_{1}, \ldots, Y_{n}\right] \rightarrow k\left[t_{1}, \ldots, t_{r}\right]
$$

by $\varphi\left(X_{i}\right)=t^{h_{i}}(1 \leq i \leq r), \varphi\left(Y_{j}\right)=t^{h_{r+j}}(1 \leq j \leq n)$,

$$
\varphi^{\prime}: k\left[X_{1}, \ldots, X_{r}, Z, Y_{r+1}, \ldots, Y_{n}\right] \rightarrow k\left[t_{1}, \ldots, t_{r}\right]
$$

by $\varphi^{\prime}\left(X_{i}\right)=t^{h_{i}}(1 \leq i \leq r), \varphi^{\prime}\left(Y_{j}\right)=t^{h_{2 r+j}}(1 \leq j \leq p-1), \varphi^{\prime}(Z)=t^{g}$.

Then, by Example 2.6 in [9], $k\left[H^{\prime}\right]\left(=\operatorname{Im} \varphi^{\prime}\right)$ is a Cohen-Macaulay ring with $\mathscr{R}_{H^{\prime}}=\mathscr{F}_{H^{\prime}}$. Also we have $k[H]$ is a Buchsbaum ring. In fact, by the choice of $a_{0}, \ldots, a_{p},(H \backslash\{0\})+H^{\prime} \subset H$. Hence, by Theorem 2.1, $k[H]$ is a Buchsbaum ring. In this case, it is easy to see that

(1) $\Delta_{H}=\left\{\left(Y_{1}, \ldots, Y_{r}\right)\right\}$

(2) $\mathscr{R}_{H}=\mathscr{F}_{H} \cup \mathscr{G}_{H}$.

\section{Codimension two Buchsbaum semigroup rings}

In this section we determine simplicial semigroups which defines Buchsbaum semigroup rings of codimension two. Henceforce we put (ht $\left.I_{H}=\right) n=2$.

When $k[H]$ is a Cohen-Macaulay ring, we determined a Gröbner bases of $I_{H}$ explicitly in Summary 2.5 of [9]. Therefore it suffices to consider Buchsbaum and not Cohen-Macaulay semigroup rings.

Then we have following result.

THEOREM 3.1. The following conditions are equivalent.

(1) $k[H]$ is a Buchsbaum ring and not Cohen-Macaulay.

(2) $\operatorname{dim} k[H]=2$ and $I_{H}$ has the following minimal basis

$$
\begin{aligned}
& Y_{i}^{b_{i}+1}-X_{1}^{a_{1}-1} X_{2}^{a_{2}+1} Y_{j}^{b_{j}-1}, Y_{1} Y_{2}-X_{1}^{a_{1}} X_{2}^{a_{2}}, \\
& Y_{j}^{b_{j}+1}-X_{1}^{a_{1}+1} X_{2}^{a_{2}-1} Y_{i}^{b_{i}-1}, X_{2} Y^{b_{j}}{ }_{i}-X_{1} X_{i}^{b_{i}}
\end{aligned}
$$

where $a_{1}, a_{2}, b_{1}, b_{2} \in \mathbf{N} \backslash\{0\},\{i, j\}=\{1,2\}$.

(3) $k[H]$ is not Cohen-Macaulay and $H$ is isomorphic to 


$$
<\left(b_{1}+b_{2}, 0\right),\left(0, b_{1}+b_{2}\right),\left(a_{1} b_{2}-1, a_{2} b_{2}+1\right),\left(a_{1} b_{1}+1, a_{2} b_{1}-1\right)>\text { as semigroup. }
$$

To prove our result, we need some preliminaries.

Now, we divide $\mathscr{F}_{H}$ into the following subsets.

$$
\begin{aligned}
& \mathscr{F}_{1}=\left\{f \in \mathscr{F}_{H} \mid \operatorname{in}(f)=Y_{1}^{b}, 0<b \in \mathbf{N}\right\} \\
& \mathscr{F}_{2}=\left\{f \in \mathscr{F}_{H} \mid \operatorname{in}(f)=Y_{2}^{c}, 0<c \in \mathbf{N}\right\} \\
& \mathscr{F}_{3}=\left\{f \in \mathscr{F}_{H} \mid \operatorname{in}(f)=Y_{1}^{b} Y_{2}^{c}, 0<b, c \in \mathbf{N}\right\}
\end{aligned}
$$

Since $\mathscr{F}_{i} \neq \phi$, there exists the minimal element of in $\left(\mathscr{F}_{i}\right)$. We denote the minimal element of in $\left(\mathscr{F}_{1}\right)$ (resp. $\mathscr{F}_{2}, \mathscr{F}_{3}$ ) by $Y_{1}^{b_{1}}$ (resp. $Y_{2}^{b_{2}}, Y_{1}^{b_{31}} Y_{2}^{b_{32}}$ ). We call $f \in$ $\mathscr{F}_{1}$ (resp. $\mathscr{F}_{2}, \mathscr{F}_{3}$ ) a minimal element of $\mathscr{F}_{1}$ (resp. $\mathscr{F}_{2}, \mathscr{F}_{3}$ ), if in $(f)=Y_{1}^{b_{1}}$ (resp. $\left.Y_{2}^{b_{2}}, Y_{1}^{b_{31}} Y_{2}^{b_{32}}\right)$.

Lemma 3.2. Suppose that $k[H]$ is a Buchsbaum ring. Then

$$
\left(\operatorname{in}\left(\mathscr{F}_{H}\right)\right)=\left(Y_{1}^{b_{1}}, Y_{2}^{b_{2}}, Y_{1}^{b_{31}} Y_{2}^{b_{32}}\right) .
$$

Proof. Since $\left(\operatorname{in}\left(\mathscr{F}_{1}\right)\right.$, in $\left.\left(\mathscr{F}_{2}\right)\right)=\left(Y_{1}^{b_{1}}, Y_{2}^{b_{2}}\right)$, it suffices to show that

$$
\operatorname{in}\left(\mathscr{F}_{3}\right) \subset\left(Y_{1}^{b_{1}}, Y_{2}^{b_{2}}, Y_{1}^{b_{31}} Y_{2}^{b_{32}}\right) .
$$

Therefore, we assume that there exists an element $f=Y_{1}^{b} Y_{2}^{b^{\prime}}-X^{\alpha} \in \mathscr{F}_{3}$ such that $Y_{1}^{b} Y_{2}^{b^{\prime}} \notin\left(Y_{1}^{b_{1}}, Y_{2}^{b_{2}}, Y_{1}^{b_{31}} Y_{2}^{b_{32}}\right)$. (Note that $b<b_{1}, b^{\prime}<b_{2}$.)

Let $f_{3}=Y_{1}^{b_{31}} Y_{2}^{b_{32}}-X^{\alpha_{3}} \in \mathscr{F}_{3}$ be a minimal element of $\mathscr{F}_{3}$. Then, by our assumption, we have either $b_{31}>b, b_{32}<b^{\prime}$ or $b_{31}<b, b_{32}>b^{\prime}$.

If $b_{31}>b$ and $b_{32}<b^{\prime}$, then we have a relation

$$
g:=Y_{1}^{b_{31}-b} f-Y_{2}^{b^{\prime}-b_{32}} f_{3}=X^{\alpha_{3}} Y_{2}^{b^{\prime}-b_{32}}-X^{\alpha} Y_{1}^{b_{31}-b} \in I_{H} .
$$

Since $Y_{1}^{b_{31}-b}, Y_{2}^{b^{\prime}-b_{32}} \notin\left(\operatorname{in}\left(\mathscr{F}_{H}\right)\right)$ and Theorem 2.6, we have in $(g) \in\left(\operatorname{in}\left(\mathscr{G}_{H}\right)\right)$.

If in $(g)=X^{\alpha_{3}} Y_{2}^{b^{\prime}-b_{32}}$, then there exists $X_{i} Y_{2}^{d}-X_{j} Y_{1}^{e} \in \mathscr{G}_{H}$ such that $X^{\alpha_{3}} Y_{2}^{b^{\prime}-b_{32}}$ is divided by $X_{i} Y_{2}^{d}$.

Since $Y_{2}^{d} \in M\left(\left[\left(x_{j}\right): \mathbf{m}\right]\right) \subset M\left(\left[\left(x_{j}\right): y_{2}\right]\right)$, we have $Y_{t}^{d+1} \in\left(\operatorname{in}\left(\mathscr{F}_{2}\right)\right)$ by Remark $1.7(2)$ and (7). Thus $Y_{2}^{b^{\prime}-b_{32}+1} \in\left(\right.$ in $\left.\left(\mathscr{F}_{2}\right)\right)$ and $b^{\prime}-b_{32}+1 \geq b_{2}$. Since $b_{32}>0$, this contradicts that $\left(b^{\prime}-b_{32}+1 \leq\right) b^{\prime}<b_{2}$. Similarly, if in $(g)=$ $X^{\alpha} Y_{1}^{b_{31}-b}$, then this contradicts that $b<b_{1}$.

When $b_{31}<b$ and $b_{32}>b^{\prime}$, it is the same way as above.

COROLlaRY 3.3 (Theorem 2.3 in [9]). The following conditions are equivalent.

(1) $k[H]$ is a Cohen-Macaulay ring of codimension two. 
(2) $I_{H}$ is generated by at most three elements.

Proof. If $k[H]$ is Cohen-Macaulay, then (in $\left(I_{H}\right)$ ) is minimally generated by at most three elements by Corollary 2.9 and Lemma 3.2. Namely, a number of minimal Gröbner bases of $I_{H}$ is at most three. Hence $\mu\left(I_{H}\right) \leq 3$.

The converse follows from Theorem 4.4 in [5].

Now, we denote the smallest element of $\sum\left(Y^{b_{1}}\right)$ (resp. $\sum\left(Y_{2}^{b_{2}}\right)$, $\left.\sum\left(Y_{1}^{b_{31}} Y_{2}^{b_{32}}\right)\right)$ by $X^{\alpha_{1}} Y_{2}^{c_{1}}$ (resp. $X^{\alpha_{2}} Y_{1}^{c_{2}}, X^{\alpha_{3}}$ ). We put

$$
\begin{gathered}
f_{1}=Y_{1}^{b_{1}}-X^{\alpha_{1}} Y_{2}^{c_{1}} \in \mathscr{F}_{1} \\
f_{2}=Y_{2}^{b_{2}}-X^{\alpha_{2}} Y_{1}^{c_{2}} \in \mathscr{F}_{2} \\
f_{3}=Y_{1}^{b_{31}} Y_{2}^{b_{31}}-X^{\alpha_{3}} \in \mathscr{F}_{3} .
\end{gathered}
$$

Remark 3.4. Since $\sum\left(X^{\alpha_{1}} Y_{2}^{c_{1}}\right)=\phi$ (resp. $\left.\sum\left(X^{\alpha_{2}} Y_{1}^{c_{2}}\right)\right)$, we have $c_{1}<b_{2}$ (resp. $c_{2}<b_{1}$ ).

Lemma 3.5. If $k[H]$ is a Buchsbaum ring and not Cohen-Macaulay, then $\operatorname{dim}(k[H])=2$ and $\mathscr{G}_{H}=\left\{X_{2} Y_{j}^{b_{j}-1}-X_{1} Y_{i}^{b_{j}-1}\right\}$ where $\{i, j\}=\{1,2\}$.

Proof. By Corollary 2.10, we have already $\operatorname{dim}(k[H])=2$. Also, by Theorem 2.6 and Definition 2.5, there exists $X_{2} Y_{j}^{d_{j}}-X_{1} Y_{i}^{d_{i}} \in \mathscr{G}_{H}$ with $d_{1}<b_{1}$, $d_{2}<b_{2}$. Since $Y_{1}^{d_{1}+1} \in\left(\operatorname{in}\left(\mathscr{F}_{H}\right)\right), d_{1}+1 \geq b_{1}$. Thus $d_{1}+1=b_{1}$. Similarly, we have $d_{2}+1=b_{2}$.

But, by (B-2) and (B-3) of Definition 2.5, if $\left(Y_{i}^{b_{i}-1}, Y_{j}^{b_{j}-1}\right) \in \Delta_{H}$, then $\left(Y_{j}^{b_{j}-1}\right.$, $\left.Y_{i}^{b_{i}-1}\right) \notin \Delta_{H}$. Hence we have $\mathscr{G}_{H}=\left\{X_{2} Y_{j}^{b_{j}-1}-X_{1} Y_{i}^{b_{i}-1}\right\}$ where $\{i, j\}=\{1,2\}$.

Lemma 3.6. For $\{i, j\}=\{1,2\}$, if there exists an element of the form

$$
X_{2}^{d_{2}} Y_{i}^{e_{i}}-X_{1}^{d_{1}} Y_{j}^{e_{j}} \in I_{H}
$$

for some $d_{1}, d_{2}, e_{1}, e_{2}>0$, then there does not exist an element of the form

$$
X_{2}^{d_{2}^{\prime}} Y_{j}^{e_{j}^{\prime}}-X_{1}^{d_{1}^{\prime}} Y_{i}^{e_{i}^{\prime}} \in I_{H}
$$

for any $d_{1}^{\prime}, d_{2}^{\prime}, e_{1}^{\prime}, e_{2}^{\prime}>0$.

Proof. Suppose that there exist $X_{2}^{d_{2}} Y_{i}^{e_{i}}-X_{1}^{d_{1}} Y_{j}^{e_{j}} \in I_{H}$ and $X_{2}^{d_{2}^{\prime}} Y_{j}^{e_{j}^{\prime}}-X_{1}^{d_{1}^{\prime}} Y_{i}^{e_{i}^{\prime}}$ $\in I_{H}$ for some $d_{1}, d_{2}, d_{1}^{\prime}, d_{2}^{\prime}, e_{1}, e_{2}, e_{1}^{\prime}, e_{2}^{\prime}>0$.

If $e_{i} \geq e^{\prime}{ }_{i}$, then we can find an element $X_{2}^{p} Y_{i}^{q}-X_{1}^{p^{\prime}} Y_{j}^{q^{\prime}} \in I_{H}$ such that 
$0<p, p^{\prime}, 0 \leq q, q^{\prime}$ and $q<e_{i}^{\prime}, q \equiv e_{i}\left(\bmod e_{i}^{\prime}\right)$ in the following manner.

We write $e_{i}=m e_{i}^{\prime}+r$, where $0<m$ and $0 \leq r<e_{i}^{\prime}$ and put $g^{\prime}=X_{2}^{d_{2}^{\prime}} Y_{j}^{e_{j}^{\prime}}-$ $X_{1}^{d_{1}^{\prime}} Y_{i}^{e_{1}^{\prime}}$. For every $0 \leq l \leq m$, we construct $g_{l}=X_{2}^{d_{2}+l d_{2}^{\prime}} Y_{i}^{e_{i}-l e_{i}^{\prime}}-X_{1}^{d_{1}+l d_{1}^{\prime}} Y_{j}^{e_{j}-e_{j}^{\prime}} \in$ $I_{H}$ as follows.

- $g_{0}=X_{2}^{b_{2}} Y_{i}^{e_{i}}-X_{1}^{d_{1}} Y_{j}^{e_{j}}$.

- Assume that $g_{0}, \ldots, g_{l}$ are constructed for $0 \leq l<m$. Then we have

$$
\begin{aligned}
& X_{1}^{d_{1}^{\prime}} g_{l}+X_{2}^{d_{2}+l d_{2}^{\prime}} Y_{i}^{e_{j}-(l+1) e_{i}^{\prime}} g^{\prime} \\
= & X_{1}^{d_{1}^{\prime}}\left(X_{2}^{d_{2}+l d_{2}^{\prime}} Y_{\imath}^{e_{i}-l e_{i}^{\prime}}-X_{1}^{d_{1}+l d_{1}^{\prime}} Y_{j}^{e_{j}-l e_{j}^{\prime}}\right)+X_{2}^{d_{2}+l d_{2}^{\prime}} Y_{i}^{e_{i}-(l+1) e_{i}^{\prime}}\left(X_{2}^{d_{2}^{\prime}} Y_{j}^{e_{j}^{\prime}}-X_{1}^{d_{1}^{\prime}} Y_{i}^{e_{i}^{\prime}}\right) \\
= & X_{2}^{d_{2}+(l+1) d_{2}^{\prime}} Y_{i}^{e_{i}-(l+1) e_{i}} Y_{j}^{e_{j}^{\prime}}-X_{1}^{d_{1}+(l+1) d_{1}^{\prime}} Y_{j}^{e_{j}-l e_{j}^{\prime}} \in I_{H} .
\end{aligned}
$$

By our assumptions (H-1) and (H-2), we have $e_{j}^{\prime} \leq e_{j}-l e_{j}^{\prime}$ and

$$
g_{l+1}:=X_{2}^{d_{2}+(l+1) d_{2}^{\prime}} Y_{i}^{e_{i}-(l+1) e_{t}^{\prime}}-X_{1}^{d_{1}+(l+1) d_{1}^{\prime}} Y_{j}^{e_{j}-(l+1) e_{j}^{\prime}} \in I_{H} .
$$

In particular, we have $g_{m}=X_{2}^{d_{2}+m d_{2}^{\prime}} Y_{i}^{r}-X_{1}^{d_{1}+m d_{1}^{\prime}} Y_{j}^{e_{j}-m e_{j}^{\prime}} \in I_{H}$.

Similarly, if $e_{\imath} \leq e_{i}^{\prime}$, then we can find an element $X_{2}^{p} Y_{\jmath}^{q^{\prime}}-X_{1}^{p^{\prime}} Y_{\imath}^{q} \in I_{H}$ such that $0<p, p^{\prime}, 0 \leq q, q^{\prime}$ and $q^{\prime}<e_{i}, q^{\prime} \equiv e_{i}\left(\bmod e_{\imath}\right)$.

Thus, by the Euclidean algorithm, we can reduce to the case $e_{i}=0$ or $e_{i}^{\prime}=0$. But, by (H-1) and (H-2), $X_{2}^{d_{2}}-X_{1}^{d_{1}} Y_{j}^{e_{j}} \notin I_{H}$ and $X_{2}^{d_{2}^{\prime}} Y_{j}^{e_{j}^{\prime}}-X_{1}^{d_{1}^{\prime}} \notin I_{H}$ for $d_{1}, d_{2}, d_{1}^{\prime}$, $d_{2}^{\prime}>0$. This is a contradiction.

Proof of Theorem 3.1. (1) $\Rightarrow(2)$. By Lemma 3.5, we have $\operatorname{dim}(k[H])=2$ and

$$
\mathscr{G}_{H}=\left\{X_{2} Y_{j}^{b_{j}-1}-X_{1} Y_{i}^{b_{i}-1}\right\}
$$

where $\{i, j\}=\{1,2\}$. We put $b_{1}^{\prime}=b_{1}-1, b_{2}^{\prime}=b_{2}-1$. Then, by Theorem 2.6 and Lemma 3.2, we have following Gröbner bases of $I_{H}$,

$$
\begin{array}{ll}
f_{1}=Y_{1}^{b_{1}^{\prime}+1}-X_{1}^{a_{11}} X_{2}^{a_{12}} Y_{2}^{c_{1}} & \in \mathscr{F}_{1} \\
f_{2}=Y_{2}^{b_{2}^{\prime}+1}-X_{1}^{a_{21}} X_{2}^{a_{22}} Y_{1}^{c_{2}} & \in \mathscr{F}_{2} \\
f_{3}=Y_{1}^{b_{31}} Y_{2}^{b_{32}}-X_{1}^{a_{1}} X_{2}^{a_{2}} & \in \mathscr{F}_{3} \\
g=X_{2} Y_{j}^{b_{j}^{\prime}}-X_{1} Y_{i}^{b_{i}^{\prime}} & \in \mathscr{G}_{H} .
\end{array}
$$

Since $Y_{1}^{b_{1}^{\prime}} \in M\left(\left[\left(x_{j}\right): \mathbf{m}\right]\right) \subset M\left(\left[\left(x_{j}\right): y_{2}\right]\right), Y_{1}^{b_{1}^{\prime}} Y_{2} \in\left(\right.$ in $\left.\left(\mathscr{F}_{H}\right)\right)$. Namely, $Y_{1}^{b_{31}} Y_{2}^{b_{32}}$ divides $Y_{1}^{b_{1}^{\prime}} Y_{2}$. Thus $b_{32}=1$. Similarly, $b_{31}=1$. Hence we have $f_{3}=Y_{1} Y_{2}-$ $X_{1}^{a_{1}} X_{2}^{a_{2}}$.

We consider the following relation 


$$
X_{2} f_{j}-Y_{j} g=X_{1} Y_{i}^{b_{i}^{\prime}} Y_{j}-X_{1}^{a_{j 1}} X_{2}^{a_{j 2}+1} Y_{i}^{c_{j}} \in I_{H}
$$

Since $c_{j}<b_{i}^{\prime}+1$ (cf. Remark 3.4), $X_{1} Y_{i}^{b_{i}^{\prime}-c_{j}} Y_{j}-X_{1}^{a_{j 1}} X_{2}^{a_{j 2}+1} \in I_{H}$. If $a_{j 1}=0$, this contradicts our assumptions $(\mathrm{H}-1)$ and (H-2). Thus $g^{\prime}=Y_{i}^{b_{i}^{\prime}-c_{j}} Y_{j}-X_{1}^{a_{j 1}-1} X_{2}^{a_{j 2}+1}$ $\in I_{H}$. By $b_{j}^{\prime}>0, Y_{j} \notin\left(\operatorname{in}\left(f_{j}\right)\right)=\left(Y_{j}^{b_{j}^{\prime}+1}\right)$ and $b_{i}^{\prime}-c_{j}>0$. Thus we have

$$
g^{\prime}-Y_{i}^{b_{i}^{\prime}-c_{j}-1} f_{3}=X_{1}^{a_{1}} X_{2}^{a_{2}} Y_{i}^{b_{i}^{\prime}-c_{j}-1}-X_{1}^{a_{j 1}-1} X_{2}^{a_{j 2}+1} \in I_{H} .
$$

Then, by (H-1) and (H-2), $Y_{i}^{b_{i}^{\prime}-c_{j}-1}-X_{1}^{a_{j 1}-1-a_{1}} X_{2}^{a_{j 2}+1-a_{2}} \in I_{H}$. But $\sum\left(Y_{i}^{b_{i}^{\prime}-c_{j}-1}\right)=\phi$. Hence $Y_{i}^{b_{i}^{\prime}-c_{j}-1}-X_{1}^{a_{j 1}-1-a_{1}} X_{2}^{a_{j 2}+1-a_{2}}=0$ and $a_{j 1}=a_{1}+1, a_{j 2}=a_{2}-1, c_{j}=b_{i}^{\prime}-$ 1. Then we have $f_{j}=Y_{j}^{b_{j}^{\prime}+1}-X_{1}^{a_{1}+1} X_{2}^{a_{2}-1} Y_{i}^{b_{i}^{\prime}-1}$.

Similarly, $f_{i}=Y_{i}^{b_{i}^{\prime}+1}-X_{1}^{a_{1}-1} X_{2}^{a_{2}+1} Y_{j}^{b_{j}^{\prime}-1}$.

Now $I_{H}$ is generated by $f_{1}, f_{2}, f_{3}, g$. Thus, by Corollary $3.3, \mu\left(I_{H}\right)=4$ and $\left\{f_{1}, f_{2}, f_{3}, g\right\}$ is a minimal basis of $I_{H}$.

$(2) \Rightarrow(3)$. Suppose that $\operatorname{dim}(k[H])=2$ and, after the permutation of variables, $I_{H}$ is minimally generated by

$$
\begin{aligned}
& f_{1}=Y_{1}^{b+1}-X_{1}^{a_{1}-1} X_{2}^{a_{2}+1} Y_{2}^{c-1} \\
& f_{2}=Y_{2}^{c+1}-X_{1}^{a_{1}+1} X_{2}^{a_{2}-1} Y_{1}^{b-1} \\
& f_{3}=Y_{1} Y_{2}-X_{1}^{a_{1}} X_{2}^{a_{2}} \\
& g=X_{2} Y_{2}^{c}-X_{1} Y_{1}^{b} .
\end{aligned}
$$

Then, by Corollary $3.3, k[H]$ is not Cohen-Macaulay.

We verify the second assertion. For $H=\sum_{i=1}^{4} \mathbf{N} h_{i}$, we put

$$
h_{1}=\left(d_{1}, 0\right), h_{2}=\left(0, d_{2}\right), h_{3}=\left(d_{31}, d_{32}\right), h_{4}=\left(d_{41}, d_{42}\right) \text {. }
$$

Since $f_{3}, g \in I_{H}$, we have $h_{3}+h_{4}=a_{1} h_{1}+a_{2} h_{2}$ and $h_{2}+c h_{4}=h_{1}+b h_{3}$. Thus $d_{4 i}=a_{i} d_{i}-d_{3 i}$ for $i=1,2$ and

$$
\begin{aligned}
& b d_{31}=c d_{41}-d_{1} \\
& b d_{32}=c d_{42}+d_{2}
\end{aligned}
$$

Solving these equations, we have

$$
\begin{aligned}
& d_{31}=\frac{d_{1}}{b+c}\left(a_{1} c-1\right) \\
& d_{32}=\frac{d_{2}}{b+c}\left(a_{2} c+1\right) \\
& d_{41}=\frac{d_{1}}{b+c}\left(a_{1} b+1\right) \\
& d_{42}=\frac{d_{2}}{b+c}\left(a_{2} b-1\right) .
\end{aligned}
$$


We define $\mathbf{Q}$-isomorphism $T: \mathbf{Q}^{2} \rightarrow \mathbf{Q}^{2}$ by $T(p, q)=(b+c)\left(\frac{p}{d_{1}}, \frac{q}{d_{2}}\right)$.

Then we have

$$
H \cong T(H)=\left\langle(b+c, 0),(0, b+c),\left(a_{1} c-1, a_{2} c+1\right),\left(a_{1} b+1, a_{2} b-1\right)\right\rangle .
$$

(3) $\Rightarrow(1)$. By the form of $H$, there exist elements

$$
\begin{aligned}
& f_{1}=Y_{1}^{b+1}-X_{1}^{a_{1}-1} X_{2}^{a_{2}+1} Y_{2}^{c-1} \\
& f_{2}=Y_{2}^{c+1}-X_{1}^{a_{1}+1} X_{2}^{a_{2}-1} Y_{1}^{b-1} \\
& f_{3}=Y_{1} Y_{2}-X_{1}^{a_{1}} X_{2}^{a_{2}} \\
& g=X_{2} Y_{2}^{c}-X_{1} Y_{1}^{b}
\end{aligned}
$$

of $I_{H}$.

CLAIM. $\mathscr{R}_{H} \backslash \mathscr{F}_{H}=\{g\}$.

Since $k[H]$ is not Cohen-Macaulay, $\mathscr{R}_{H} \backslash \mathscr{F}_{H} \neq \phi$ (cf. Corollary 2.9). Thus $L:=\mathscr{R}_{H} \backslash \mathscr{F}_{H} \cup\{g\}$ is not empty, if $\mathscr{R}_{H} \backslash \mathscr{F}_{H} \neq\{g\}$.

If there exists $g^{\prime} \in L$, then, by Lemma 3.6, we can write $g^{\prime}=X_{2}^{d_{2}} Y_{2}^{e_{2}}-$ $X_{1}^{d_{1}} Y_{1}^{e_{1}}$ where $0<d_{1}, d_{2}, e_{1}, e_{2}$. Since $Y_{1}^{b+1}, Y_{2}^{c+1} \in\left(\operatorname{in}\left(I_{H}\right)\right)$ and $Y_{1}^{e_{1}}, Y_{2}^{e_{2}} \notin$ (in $\left.\left(I_{H}\right)\right), e_{1} \leq b$ and $e_{2} \leq c$. Then we have

$$
Y_{2}^{c-e_{2}} g^{\prime}-X_{2}^{d_{2}-1} g=X_{1} Y_{1}^{e_{1}}\left(X_{2}^{d_{2}-1} Y_{1}^{b-e_{1}}-X_{1}^{d_{1}-1} Y_{2}^{c-e_{2}}\right) \in I_{H} .
$$

Hence we have $g_{1}:=X_{2}^{d_{2}-1} Y_{1}^{b-e_{1}}-X_{1}^{d_{1}-1} Y_{2}^{c-e_{2}} \in I_{H}$. Note that $g_{1} \neq 0$ (since $g^{\prime} \neq g$ ). Then, by $(\mathrm{H}-1)$ and (H-2), either $b-e_{1}>0$ or $c-e_{2}>0$.

If $b-e_{1}>0$, then we have

$$
X_{1}^{d_{1}} g_{1}-X_{2}^{d_{2}-1} Y_{1}^{b-2 e_{1}} g^{\prime}=X_{2}^{2 d_{2}-1} Y_{1}^{b-2 e_{1}} Y_{2}^{e_{2}}-X_{1}^{2 d_{1}-1} Y_{2}^{c-e_{2}} \in I_{H} .
$$

Since $d_{1}, d_{2}>0$, we have $2 d_{1}-1$ and $2 d_{2}-1>0$. Also, by $(\mathrm{H}-1)$ and $(\mathrm{H}-2)$, $e_{2} \leq c-e_{2}$. Thus $0 \neq X_{2}^{2 d_{2}-1} Y_{1}^{b-2 e_{1}}-X_{1}^{2 d_{1}-1} Y_{2}^{c-2 e_{2}} \in I_{H}$. But this contradicts Lemma 3.6.

If $c-e_{2}>0$, we have a contradiction in the same way as above. Hence $\mathscr{R}_{H}=$ $\mathscr{F}_{H} \cup\{g\}$.

The proof of Claim is completed.

By Theorem 2.6 and Claim, it suffices to show that $\left(Y_{1}^{b}, Y_{2}^{c}\right) \in \Delta_{H}$. Since we have relations $f_{1}, f_{2}, f_{3} \in I_{H}\left(Y_{1}^{b}, Y_{2}^{c}\right)$ satisfies condition (B-2). Also it is clear that $\left(Y_{1}^{b}, Y_{2}^{c}\right)$ satisfies conditions (B-1), (B-4) and (B-5). Then we have only to prove that $\left(Y_{1}^{b}, Y_{2}^{c}\right)$ satisfies condition $(\mathrm{B}-3)$.

If $Y_{1}^{b} \in M\left(\left[\left(x_{1}\right): \mathbf{m}\right]\right) \subset M\left(\left[\left(x_{1}\right): x_{2}\right]\right)$, then we have $X_{2} Y_{1}^{b}-X_{1}^{d_{1}} X_{2}^{d_{2}} Y_{1}^{e_{1}} Y_{2}^{e_{2}}$ $\in I_{H}$ with $\sum\left(Y_{1}^{e_{1}} Y_{2}^{e_{2}}\right)=\phi$ and $d_{1}>0$. Since $\sum\left(Y_{1}^{b}\right)=\phi, d_{2}=0$ and, by $(\mathrm{H}-1)$ 
and (H-2), $e_{1}<b$. Then we have $X_{2} Y_{1}^{b-e_{1}}-X^{X^{d_{1}}} Y_{2}^{e_{2}} \in I_{H}$. But, by Lemma 3.6, this is a contradiction. Hence we have $Y_{1}^{b} \notin M\left(\left[\left(x_{1}\right): \mathbf{m}\right]\right)$. Similarly, $Y_{2}^{c} \notin M\left(\left[\left(x_{2}\right)\right.\right.$ : m]).

Example 3.7. Let $0<a<b<c \in \mathbf{N}$. We consider the following simplicial semigroup

$$
H=\langle(c, 0),(0, c),(c-b, b),(c-a, a)\rangle .
$$

and the semigroup ring $k[H]=k\left[t_{1}^{c}, t_{2}^{c}, t_{1}^{c-b} t_{2}^{b}, t_{1}^{c-a} t_{2}^{a}\right]$.

In [3], H. Bresinsky, P. Schenzel and W. Vogel discussed arithmetical Buchsbaum curves in $\mathbf{P}_{k}^{3}$ and showed that $k[H]$ is a Buchsbaum ring and not Cohen-Macaulay if and only if

$$
H \cong\langle(4 m, 0),(0,4 m),(2 m-1,2 m+1),(2 m+1,2 m-1)\rangle
$$

for some $m>0$ (cf. Theorem 3 in [3]).

We can verify this fact as follows.

By Theorem 3.1, if $k[H]$ is a Buchsbaum ring and not Cohen-Macaulay, then $I_{H}$ has the following minimal basis

$$
\begin{aligned}
& f_{1}=Y_{1}^{b_{1}+1}-X_{1}^{a_{1}-1} X_{2}^{a_{2}+1} Y_{2}^{b_{2}-1} \\
& f_{2}=Y_{2}^{b_{2}+1}-X_{1}^{a_{1}+1} X_{2}^{a_{2}-1} Y_{1}^{b_{1}-1} \\
& f_{3}=Y_{1} Y_{2}-X_{1}^{a_{1}} X_{2}^{a_{2}} \\
& g=X_{2} Y_{2}^{b_{2}}-X_{1} Y_{1}^{b_{1}},
\end{aligned}
$$

where $a_{1}, a_{2}, b_{1}, b_{2} \in \mathbf{N} \backslash\{0\}$. In this case, these are homogeneous polynomials with respect to the total degree. Then we have $a_{1}=a_{2}=1, b_{1}=b_{2}$ and

$$
H \cong\left\langle\left(2 b_{1}, 0\right),\left(0,2 b_{1}\right),\left(b_{1}-1, b_{1}+1\right),\left(b_{1}+1, b_{1}-1\right)\right\rangle .
$$

If $b_{1}=2 m+1(m \geq 0)$, then $Y_{1}^{2 m+1}-X_{1}^{m} X_{2}^{m+1} \in I_{H}$. This contradicts that $\left\{f_{1}, f_{2}, f_{3}, g\right\}$ is a Gröbner bases of $I_{H}$ (cf. proof of Theorem 3.1). Hence we have $b_{1}=2 m(m>0)$ and

$$
H \cong\langle(4 m, 0),(0,4 m),(2 m-1,2 m+1),(2 m+1,2 m-1)\rangle .
$$

Also $I_{H}$ is generated by

$$
\begin{aligned}
& f_{1}=Y_{1}^{2 m+1}-X_{2} Y_{2}^{2 m-1} \\
& f_{2}=Y_{1}^{2 m+1}-X_{1} Y_{2}^{2 m-1} \\
& f_{3}=Y_{1} Y_{2}-X_{1} X_{2} \\
& g=X_{2} Y_{2}^{2 m}-X_{1} Y_{1}^{2 m}
\end{aligned}
$$


Conversely, for $H=\langle(4 m, 0),(0,4 m),(2 m-1,2 m+1),(2 m+1,2 m$ $-1)\rangle$, it is easy to see that $k[H]$ is not Cohen-Macaulay (cf. Theorem 3.8 in [9]). Hence, by Theorem $3.1(3), k[H]$ is a Buchsbaum ring.

Acknowledgement. I would like to thank Professor K. Watanabe and members of Professor Goto's seminar in Meiji University for many helpful conversation.

\section{REFERENCES}

[1] H. Bresinsky, Symmetric semigroups of integers generated by 4 elements, Manuscripta Math., 17 (1975), 205-219.

[2] - Minimal free resolutions of monomial curves in $\mathbf{P}_{k}^{3}$, Linear Algebra and Its Applications, 59 (1984), 121-129.

[ 3 ] - P. Schenzel and W. Vogel, On liaison, arithemetical Buchsbaum curves and monomial curves in $\mathbf{P}^{3}$, J. Algebra, 86 (1984), 283-301.

[4] B. Buchberger, Gröbner bases: An algorithmic method in polynomial ideal theory, In "Multidimensional system theory" (N. K. Bose ed.), 184-232, Reidel Publ. Comp., 1985.

[5] E. G. Evans and P. Griffith, Syzygies, London, Math. Soc. Lect. Notes Series no. 106, Camb. Univ. Press, 1985.

[6] S. Goto, Cohen-Macaulayfication of certain Buchsbaum ring, Nagoya Math. J., 80 (1980), 107-116.

[ 7 ] S. Goto, N. Suzuki and K. Watanabe, On affine semigroup rings, Japan, J. Math., 2 (1976), 1-12.

[8] J. Herzog, Generators and relations of abelian semigroups and semigroup rings, Manuscripta Math., 3 (1970), 175-193.

[9] Y. Kamoi, Defining ideals of Cohen-Macaulay semigroup rings, Comm. in Algebra, 20 (1992), 3163-3189.

[10] L. Robbiano, Introduction to the theory of Gröbner basis, Queen's Papers in Pure and Applied Maths no. 80 vol. 5, 1988.

[11] L. Robbiano and G. Valla, Some curves in $\mathbf{P}^{3}$ are set-theoretic complete intersections, In "Algebraic Geometry-Open Problems", Lect. Notes in Math. 997, Springer, 391-399, 1983.

[12] J. Stücrad and W. Vogel, Buchsbaum rings and applications, Springer, Berlin, 1986.

Department of Mathematics

Tokyo Metropolitan University

Minami-Ohsawa 1-1, Hachioji-shi

Tokyo, 192-03 Japan 